\title{
Power Compact Disjointness Preserving Operators on Continuous Functions
}

\author{
YING-FEN LIN AND NGAI-CHING WONG
}

\begin{abstract}
Let $T$ be a disjointness preserving bounded linear operator on $C_{0}(X)$, where $X$ is a locally compact Hausdorff space. We give a sufficient and necessary condition for $T$ being power compact. Moreover, a description of the eigenvalues and eigenfunctions of such $T$ is given.
\end{abstract}

\section{INTRODUCTION}

Let $X$ be a locally compact Hausdorff space, and $C_{0}(X)$ be the Banach space of continuous (real or complex) functions on $X$ vanishing at infinity. A linear operator $T$ from $C_{0}(X)$ into $C_{0}(Y)$ is disjointness preserving if $T f \cdot T g=0$ in $C_{0}(Y)$ whenever $f \cdot g=0$ in $C_{0}(X)$. In [1], Abramovich developed the basic theory of such operators between general vector lattices. Lately such operators were studied in the space of real or complex-valued continuous functions. It is shown that a disjointness preserving linear operator $T: C_{0}(X) \rightarrow C_{0}(Y)$ is exactly a weighted composition operator $T f=h \cdot f \circ \varphi$ for some map $\varphi$ from $Y$ into $X$ and scalar-valued function $h$ on $Y$ (see also [4, 5]).

Let $k$ be a positive integer; then a real or complex linear operator $T$ on $C_{0}(X)$ is called $k$-compact if $T^{k}$ is compact and is said to be power compact if it is $k$-compact for some $k$. In this paper, we shall give a sufficient and necessary condition for bounded disjointness preserving linear operators on continuous functions being power compact. Moreover, we develop a spectral theory of power compact disjointness preserving bounded linear operators.

We would like to express our gratitude to Anthony Lau for many useful comments.

2000 Mathematics Subject Classification. 47B33, 47B38, 46J10.

Key words and phrases. Disjointness preserving operators, compact operators, power compact operators, spectral theory. 


\section{Power Compact Disjointness Preserving Operators}

Let $X$ be a locally compact Hausdorff space of infinite cardinality. Note that a power compact operator might not be bounded. In [4], it is shown that there is always an unbounded disjointness preserving linear operator from $C_{0}(X)$ onto an infinite-dimensional subspace of $C_{0}(Y)$ provided $X$ and $Y$ are infinite sets. There is also a comprehensive description of unbounded disjointness preserving linear functionals in [3]. In the following, we present an easy construction of such a functional, and also an example of unbounded power compact disjointness preserving linear operators. Unlike the bounded ones, unbounded power compact disjointness preserving linear operators might not have a nice structure.

Lemma 2.1. Let $X$ be a locally compact Hausdorff space of infinite cardinality. Then there is always an unbounded disjointness preserving linear functional of $C_{0}(X)$.

Proof. For each point $x$ in $X \cup\{\infty\}$, let $I_{x}$ (resp. $M_{x}$ ) be the ideal of $C_{0}(X)$ consisting of all functions vanishing in a neighborhood of $x$ (resp. vanishing at $x$ ). It is not difficult to see that there exists some $x$ in $X \cup\{\infty\}$ with $I_{x} \varsubsetneqq M_{x}$ (see [3, Proposition 2.6]). Since $I_{x}$ is the intersection of all prime ideals of $C_{0}(X)$ containing it, there is a prime ideal $P$ of $C_{0}(X)$ such that $I_{x} \varsubsetneqq P \varsubsetneqq M_{x}$. Choose any linear lifting $\Phi$ to $C_{0}(X)$ of a linear functional of $C_{0}(X) / P$ such that $\Phi(k)=1$ for some $k$ in $M_{x}$. In this way, we obtain an unbounded disjointness preserving linear functional $\Phi$ of $C_{0}(X)$. Indeed, if $f$ and $g$ in $C_{0}(X)$ have a zero product, since $P$ is prime, one of $f$ and $g$ belongs to $P$. As $\left.\Phi\right|_{P}=0$, one of $\Phi(f)$ and $\Phi(g)$ must be zero.

Example 2.2. Let $X=[0,1] \cup\{2\} \subset \mathbb{R}$ and $\Phi: C[0,1] \rightarrow \mathbb{C}$ be an unbounded disjointness preserving linear functional such that $\Phi\left(1_{[0,1]}\right)=1$, where $1_{[0,1]}$ is the constant one function on $[0,1]$ (see Lemma 2.1). Let $T: C(X) \rightarrow C(X)$ be defined by

$$
\left.T f\right|_{[0,1]}=f(0) 1_{[0,1]} \quad \text { and } \quad T f(2)=\Phi\left(\left.f\right|_{[0,1]}\right) .
$$

Then $T$ is an unbounded 2-compact disjointness preserving linear operator. Indeed,

$$
\left.T^{2}(f)\right|_{[0,1]}=f(0) 1_{[0,1]} \quad \text { and } \quad T^{2}(f)(2)=\Phi\left(f(0) 1_{[0,1]}\right)=f(0) .
$$

Thus $T^{2}$ is of rank one. 
In the following, all power compact linear operators are assumed to be bounded.

Theorem 2.3 ([7, Theorem 2.8]). Let $T: C_{0}(X) \rightarrow C_{0}(Y)$ be a disjointness preserving bounded linear operator. Then $T$ is compact if and only if there is a sequence $\left\{x_{n}\right\}_{n}$ of distinct points in $X$ and a norm null mutually disjoint sequence $\left\{h_{n}\right\}_{n}$ in $C_{0}(Y)$ such that

$$
T f=\sum_{n} f\left(x_{n}\right) h_{n} \quad \text { for all } f \in C_{0}(X) .
$$

In general, for a disjointness preserving bounded linear operator $T$ on $C_{0}(X)$, let $\delta_{x}$ be the point mass at $x$ in $X \cup\{\infty\}$ and thus $\delta_{x} \circ T(f)=T f(x)$ for all $f$ in $C_{0}(X)$. Set

$$
X_{\infty}=\left\{x \in X \cup\{\infty\}: \delta_{x} \circ T=0\right\} \quad \text { and } \quad X^{\prime}=X \backslash X_{\infty} .
$$

There exist a continuous function $\varphi$ from $X^{\prime}$ to $X$ and a nonvanishing continuous function $h$ on $X^{\prime}$ such that

$$
\left.T f\right|_{X_{\infty}}=0 \text { and }\left.T f\right|_{X^{\prime}}=h \cdot f \circ \varphi .
$$

See, e.g., $[5,7]$.

We extend $\varphi$ and $h$ in (2.2) to the not necessarily continuous functions $\tilde{\varphi}$ and $\tilde{h}$ on the whole of $X \cup\{\infty\}$ by setting $\tilde{\varphi} \equiv \infty$ and $\tilde{h} \equiv 0$ outside $X^{\prime}$. In this way, we can write

$$
T f=\tilde{h} \cdot f \circ \tilde{\varphi} .
$$

Theorem 2.4. Let $T$ be a disjointness preserving linear operator on $C_{0}(X)$ carrying the form (2.3) and $k$ be a positive integer. Then $T$ is $k$-compact if and only if

(1) the image of the kth power $\tilde{\varphi}^{k}$ of $\tilde{\varphi}$ is a countable set $\tilde{\varphi}^{k}(X \cup$ $\{\infty\})=\left\{\infty, x_{1}, x_{2}, \ldots\right\}$ such that $X_{n}=\tilde{\varphi}^{-k}\left(x_{n}\right)$ is open in $X$ for each $n=1,2, \ldots ;$ and

(2) the function $\tilde{h} \cdot \tilde{h} \circ \tilde{\varphi} \cdots \tilde{h} \circ \tilde{\varphi}^{k-1}$ is continuous on $X \cup\{\infty\}$.

Proof. Observe that

$$
T^{k} f=\left(\tilde{h} \cdot \tilde{h} \circ \tilde{\varphi} \cdots \tilde{h} \circ \tilde{\varphi}^{k-1}\right) \cdot f \circ \tilde{\varphi}^{k} .
$$

The necessity follows from Theorem 2.3. We verify the sufficiency. Denote $\tilde{h} \cdot \tilde{h} \circ \tilde{\varphi} \cdots \tilde{h} \circ \tilde{\varphi}^{k-1}$ by $\Psi$ for convenience. For each $n$, let $\Psi_{n}=$ $\Psi \chi_{X_{n}}$, where $\chi_{X_{n}}$ is the characteristic function of $X_{n}$. Then $\Psi_{n}$ is continuous on $X \cup\{\infty\}$ and $T^{k} f=\sum_{n} f\left(x_{n}\right) \Psi_{n}$ pointwise on $X$. We claim that the sum $T^{k} f=\sum_{n} f\left(x_{n}\right) \Psi_{n}$ converges uniformly. 
Let $\varepsilon>0$ and observe that the set $\left\{x \in X^{\prime}:|\Psi(x)| \geq \varepsilon\right\}$ is a compact subset of $X^{\prime}=\bigcup_{n} X_{n}$. Hence, there is a positive integer $N$ such that $|\Psi(x)|<\varepsilon$ whenever $x \notin \bigcup_{n=1}^{N} X_{n}$. In other words, $\left\|\Psi_{n}\right\|<\varepsilon$ for all $n>N$. Thus the sum converges uniformly, and so $T^{k}$ is compact.

Let $T=\sum_{n} \delta_{x_{n}} \otimes h_{n}$, that is, $T f=\sum_{n} f\left(x_{n}\right) h_{n}$, be a compact disjointness preserving linear operator on $C_{0}(X)$. Note that for each $n$, we have $h_{m}\left(x_{n}\right) \neq 0$ for at most one $m$. A finite set $\left\{x_{m_{1}}, x_{m_{2}}, \ldots, x_{m_{n}}\right\}$ is said to be a primitive cycle for $T$ and denoted by $\left[x_{m_{1}} ; x_{m_{2}} ; \ldots ; x_{m_{n}}\right]$ if

$$
h_{m_{n}}\left(x_{m_{n-1}}\right) \cdots h_{m_{3}}\left(x_{m_{2}}\right) h_{m_{2}}\left(x_{m_{1}}\right) h_{m_{1}}\left(x_{m_{n}}\right) \neq 0 \text {. }
$$

Theorem 2.5 ([7, Theorem 4.7]). Let $T$ be a compact disjointness preserving complex linear operator on $C_{0}(X)$ of the form $T=$ $\sum_{n} \delta_{x_{n}} \otimes h_{n}$. A nonzero complex number $\lambda$ is an eigenvalue of $T$ if and only if there is a primitive cycle $\left[x_{m_{1}} ; x_{m_{2}} ; \ldots ; x_{m_{n}}\right]$ for $T$ such that

$$
\lambda^{n}=h_{m_{n}}\left(x_{m_{n-1}}\right) \cdots h_{m_{3}}\left(x_{m_{2}}\right) h_{m_{2}}\left(x_{m_{1}}\right) h_{m_{1}}\left(x_{m_{n}}\right) .
$$

A nonzero eigenfunction $f$ of $T$ associated with $\lambda$ can be constructed as

$$
f=\left[I+\sum_{k \geq 1} \frac{T^{k-1}(T-\lambda)}{\lambda^{k}}\right] f^{\prime}
$$

where

$$
\begin{aligned}
f^{\prime}=\frac{h_{m_{n}}\left(x_{m_{n-1}}\right) \cdots h_{m_{3}}\left(x_{m_{2}}\right) h_{m_{2}}\left(x_{m_{1}}\right)}{\lambda^{n-1}} h_{m_{1}} \\
\quad+\frac{h_{m_{n}}\left(x_{m_{n-1}}\right) \cdots h_{m_{3}}\left(x_{m_{2}}\right)}{\lambda^{n-2}} h_{m_{2}}+\cdots+h_{m_{n}} .
\end{aligned}
$$

All such eigenfunctions $f$ generate the eigenspace of $T$ for $\lambda$.

Recall that all nonzero spectral values of a power compact operator on a Banach space are eigenvalues (see, e.g., [6, p. 241]).

Theorem 2.6. Let $T$ be a $k$-compact disjointness preserving complex linear operator on $C_{0}(X)$. Write $T^{k} f=\sum_{n} f\left(x_{n}\right) h_{n}$ as in (2.1). Then for each nonzero eigenvalue $\lambda$ of $T$, there is a primitive cycle $\left[x_{m_{1}} ; x_{m_{2}} ; \ldots ; x_{m_{n}}\right]$ for $T^{k}$ such that

$$
\lambda^{k n}=h_{m_{n}}\left(x_{m_{n-1}}\right) \cdots h_{m_{3}}\left(x_{m_{2}}\right) h_{m_{2}}\left(x_{m_{1}}\right) h_{m_{1}}\left(x_{m_{n}}\right) .
$$


Conversely, suppose a nonzero complex number $\lambda$ satisfies (2.4) for some primitive cycle $\left[x_{m_{1}} ; x_{m_{2}} ; \ldots ; x_{m_{n}}\right]$. Then there exists at least one kth root $\omega$ of the unity such that $\omega \lambda$ is an eigenvalue of $T$.

Proof. If $\lambda \neq 0$ is an eigenvalue of $T$ with an eigenfunction $f$, then $T f=\lambda f$ and $T^{k} f=\lambda^{k} f$. Note that $T^{k}$ is a compact disjointness preserving linear operator on $C_{0}(X)$. By Theorem 2.5, there is a primitive cycle $\left[x_{m_{1}} ; x_{m_{2}} ; \ldots ; x_{m_{n}}\right]$ for $T^{k}$ satisfying (2.4).

Conversely, for each nonzero $\lambda$ in $\mathbb{C}$ with $\lambda^{k n}$ satisfying (2.4), we shall prove that $\omega^{j} \lambda$ is in $\sigma(T)$ for some $j=1,2, \ldots, k$, where $\omega=e^{2 \pi i / k}$. Suppose this were not true, then $T-\omega^{j} \lambda I$ is invertible for all $j=1,2, \ldots, k$. However, this would provide a contradiction that

$$
T^{k}-\lambda^{k} I=\prod_{j=1}^{k}\left(T-\omega^{j} \lambda I\right)
$$

is invertible, when $\lambda^{k}$ is an eigenvalue of $T^{k}$ by Theorem 2.5.

As indicated in the proof of Theorem 2.6, every eigenfunction of $T$ associated with a nonzero eigenvalue $\lambda$ is an eigenfunction of $T^{k}$ associated with $\lambda^{k}$. Although the converse is not too complicated either, we shall see the second example in the following that some careful analysis might be needed. The third one explains that nothing can be done for those eigenfunctions associated with the zero eigenvalue.

Example 2.7. (a) Let $X=(0,2) \cup(3,5) \subset \mathbb{R}$ and $T: C_{0}(X) \rightarrow$ $C_{0}(X)$ be the compact disjointness preserving linear operator defined by

$$
T f=f(1) \chi_{(0,2)}+f(4) \chi_{(3,5)} .
$$

We have $T^{2} f=f(1) \chi_{(0,2)}+f(4) \chi_{(3,5)}=T f$. Thus, $\sigma(T)=$ $\sigma\left(T^{2}\right)=\{0,1\}$. Note that -1 does not belong to $\sigma(T)$.

(b) Let $X=[-2,-1] \cup[1,2] \subset \mathbb{R}$ and $T: C(X) \rightarrow C(X)$ be defined by

$$
T f(x)=f(1) h_{1}(x)+f(-x) h_{2}(x) \quad \text { for all } \quad x \in X,
$$

where $h_{1}$ and $h_{2}$ are the characteristic functions of the intervals $[-2,-1]$ and $[1,2]$, respectively. Then, $T^{2} f=f(-1) h_{1}+f(1) h_{2}$ is a compact endomorphism of $C(X)$. By a direct computation, we see that $\sigma(T)=\{-1,0,1\}$ and $\sigma\left(T^{2}\right)=\{0,1\}$. The 
eigenspace of $T$ for the eigenvalue 1 is spanned by $h_{1}+h_{2}$ and the one for -1 is spanned by $h_{1}-h_{2}$, while the eigenspace of $T^{2}$ for 1 is two-dimensional and spanned by $h_{1}$ and $h_{2}$. In particular, neither $h_{1}$ nor $h_{2}$ is an eigenfunction of the 2-compact lattice endomorphism $T$.

(c) Let $X=(-1,0) \cup(0,1) \subset \mathbb{R}$ and $T: C_{0}(X) \rightarrow C_{0}(X)$ be defined by

$$
T f(x)= \begin{cases}f(-x) \sin \frac{1}{x}, & 0<x<1 ; \\ 0, & -1<x<0 .\end{cases}
$$

Then $T$ has zero square and, in particular, is 2-compact. Hence $\sigma(T)=\sigma\left(T^{2}\right)=\{0\}$. However, every $f$ in $C_{0}(X)$ non-vanishing on $(-1,0)$ is an eigenfunction of $T^{2}$ but not of $T$. We also note that in this case $\tilde{\varphi}^{2}(X \cup\{\infty\})=\{\infty\}$ and $\tilde{h} \cdot \tilde{h} \circ \tilde{\varphi}=0$, while neither the range of $\tilde{\varphi}$ is countable nor $\tilde{h}$ is continuous on $X \cup\{\infty\}$.

For $\theta_{0}, \theta_{1} \in[0,2 \pi)$, we call the subset

$$
\Delta\left(\theta_{0}, \theta_{1}\right)=\left\{r e^{i \theta}: r \geq 0, \theta_{0} \leq \theta<\theta_{0}+\theta_{1}\right\}
$$

of $\mathbb{C}$ a sector with angle $\theta_{1}$. In $[2,8]$, disjointness preserving endomorphisms of Banach lattices with spectra contained in a sector were discussed. In the following, we see that the spectral theory of $k$ compact disjointness preserving bounded linear operators on $C_{0}(X)$ with spectrum contained in a sector with angle not greater than $\pi / k$ is quite simple.

Theorem 2.8. Let $T: C_{0}(X) \rightarrow C_{0}(X)$ be a compact disjointness preserving linear operator with spectrum contained in a sector with angle not greater than $\pi$. Suppose $T$ carries the form $T f=$ $\sum_{n} f\left(x_{n}\right) h_{n}$ for all $f \in C_{0}(X)$. Then the spectrum of $T$ is

$$
\sigma(T)=\left\{0, h_{1}\left(x_{1}\right), h_{2}\left(x_{2}\right), \ldots\right\} .
$$

Moreover, associated with each nonzero $h_{n}\left(x_{n}\right)$ in $\sigma(T)$ an eigenfunction of $T$ is of the form

$$
f_{n}=\left[I+\sum_{k \geq 1} \frac{T^{k-1}\left(T-h_{n}\left(x_{n}\right)\right)}{h_{n}\left(x_{n}\right)^{k}}\right] h_{n} .
$$

The finite dimensional eigenspace of $T$ associated with a nonzero eigenvalue $h_{n}\left(x_{n}\right)$ is spanned by all such $f_{n}$. 
Proof. Suppose $\left[x_{j_{1}} ; x_{j_{2}} ; \ldots ; x_{j_{l}}\right]$ is a primitive cycle for $T$. By Theorem 2.5 , each $l$ th root of the nonzero product

$$
h_{j_{l}}\left(x_{j_{l-1}}\right) \cdots h_{j_{3}}\left(x_{j_{2}}\right) h_{j_{2}}\left(x_{j_{1}}\right) h_{j_{1}}\left(x_{j_{l}}\right)
$$

is an eigenvalue of $T$. Since $\sigma(T)$ is contained in a sector with angle not greater than $\pi$, we have $l=1$. Then the proof is finished by Theorem 2.5.

Corollary 2.9. Let $T$ be a $k$-compact disjointness preserving bounded linear operator on $C_{0}(X)$ with spectrum contained in a sector $\Delta\left(\theta_{0}, \theta_{1}\right)$ with angle $\theta_{1}$ not greater than $\pi / k$. A nonzero $\lambda \in \Delta\left(\theta_{0}, \theta_{1}\right)$ is an eigenvalue of $T$ if and only if $\lambda^{k}$ is an eigenvalue of $T^{k}$. Moreover, the eigenspace of $T$ for $\lambda$ coincides with that of $T^{k}$ for $\lambda^{k}$.

Corollary 2.10. Let $T$ be a $k$-compact disjointness preserving bounded linear operator on $C_{0}(X)$ with positive spectrum. Suppose $T^{k} f=$ $\sum_{n} f\left(x_{n}\right) h_{n}$ for all $f$ in $C_{0}(X)$. Then all functions $h_{n} \geq 0$, and $\sigma(T)=\left\{0, h_{1}\left(x_{1}\right)^{1 / k}, h_{2}\left(x_{2}\right)^{1 / k}, \ldots\right\}$. An eigenfunction $f_{n}$ of $T$ for each positive eigenvalue $h_{n}\left(x_{n}\right)^{1 / k}$ is given by

$$
f_{n}=\left[I+\sum_{l \geq 1} \frac{T^{(l-1) k}\left(T^{k}-h_{n}\left(x_{n}\right)^{1 / k}\right)}{h_{n}\left(x_{n}\right)^{l / k}}\right] h_{n} .
$$

All such $f_{n}$ span the finite-dimensional eigenspace of $T$ for a given positive eigenvalue $\lambda$ with $\lambda^{k}=h_{n}\left(x_{n}\right)$.

\section{REFERENCES}

[1] Yu. A. Abramovich, Multiplicative representation of disjointness preserving operators, Indag. Math. 45 (1983), 265-279.

[2] W. Arendt, Spectral properties of Lamperti operators, Indiana Univ. Math. J. 32 (1983), 199-215.

[3] L. G. Brown and N.-C. Wong, Unbounded disjointness preserving linear functionals, Monatsh. Math. 141 (2004), 21-32.

[4] K. Jarosz, Automatic continuity of separating linear isomorphisms, Canad. Math. Bull. 33 (1990), 139-144.

[5] J.-S. Jeang and N.-C. Wong, Weighted composition operators of $C_{0}(X)$ 's, J. Math. Anal. Appl. 201 (1996), 981-993.

[6] P. D. Lax, Functional Analysis, Wiley-Interscience, New York, 2002.

[7] Y.-F. Lin and N.-C. Wong, Compact disjointness preserving operators of continuous functions, preprint.

[8] X.-D. Zhang, Decomposition theorems for disjointness preserving operators, J. Funct. Anal. 116 (1993), 158-178. 
Ying-Fen Lin,

Department of Mathematics Education,

National Hualien University of Education,

Hua-Lien 970,

Taiwan, R.O.C.

linyf@mail.nhlue.edu.tw

Ngai-Ching Wong,

Department of Applied Mathematics,

National Sun Yat-sen University,

Kaohsiung 804,

Taiwan, R.O.C.

wong@math.nsysu.edu.tw

Received in final form on 25 July 2005. 